\title{
A HYBRID APPROACH USING FORECASTING AND DISCRETE-EVENT SIMULATION FOR ENDOSCOPY SERVICES
}

\author{
Alison Harper \\ Navonil Mustafee \\ The Business School \\ University of Exeter \\ Streatham Court, Rennes Drive \\ Exeter EX4 4ST, UK
}

\author{
Mark Feeney \\ Endoscopy Unit \\ Torbay \& South Devon NHS Trust \\ Lowes Bridge \\ Torquay TQ2 7AA, UK
}

\begin{abstract}
Healthcare services worldwide are prioritizing efficiency of delivery and optimization of resource allocation. Efficient healthcare delivery relies on the coordination of demand and capacity, but forecasting studies often predict demand without regard for future capacity constraints. Likewise, capacity planning requires strategic decision-making, therefore planning tools should allow decision-makers to examine the consequences of changing demand and likely capacities required over time. The aim of the study is to evaluate a hybrid methodology using discrete-event simulation and demand forecasting in the healthcare domain. A case-study investigates the application of official population projections with local historical demand data to forecast demand for a healthcare diagnostic service. The resultant forecasts are then used with DES in a hybrid systems modeling approach. This provides plausible demand forecasts for future capacity planning and resource allocation in a preventative healthcare service. It also contributes to debates on the value of hybrid approaches in supporting real-world decision-making.
\end{abstract}

\section{INTRODUCTION}

This paper employs a case study of a healthcare diagnostic service to evaluate a hybrid application of simulation and forecasting. Applications of hybrid simulation methodologies claim to increase insights into a problem situation (Fakhimi, Mustafee, and Stergioulas 2016; Eldabi et al. 2016), and are becoming an important research area within the field of modeling and simulation (M\&S). Powell and Mustafee (2016) aimed to provide a cohesive definition of hybrid methodologies, by distinguishing between hybrid simulation, where two or more simulation techniques have been integrated at the model implementation stage, and hybrid systems modeling, where inter-disciplinary approaches and techniques, including those from Operations Research, are used with computer simulation. This wider definition of hybrid simulation has also been expounded by one of the panelists in the panel paper by Eldabi et al. (2016), who described models that are developed by combining the methodological strengths of individual modeling techniques with methods from other disciplines or the wider field of OR. They term these hybrid studies a hybrid systems modeling approach or hybrid approach (HA).

Discrete-event simulation (DES) models the movement of individual entities through a system described by a series of queues and servers. It allows researchers to evaluate strategies for capacity planning and resource allocation, and has been used extensively in healthcare to support more efficient and informed decision-making (Katsaliaki and Mustafee 2011; Brailsford et al. 2009). Worldwide shifts in population demographics translates to an increase in demand for healthcare services; in a publicly-funded healthcare system, such as the UK NHS, this demand will have to be met with increasingly constrained budgets. Efficiency of delivery of healthcare services relies on the coordination of demand and capacity, but forecasting studies often predict demand without regard for future capacity constraints. Likewise, capacity 
planning requires strategic decision-making, therefore planning tools should allow decision-makers to examine the consequences of changing demand and likely capacities required over time (Harper 2002). Demand forecasting combined with simulation can potentially address both of these requirements.

The aim of this paper is to evaluate how forecasting methods might be used with DES in a HA, using endoscopy diagnostic services in one Clinical Commissioning Group (CCG) in the UK as a case study. This demonstrates the applicability of a HA in a real-life setting to inform service planning at the local (CCG) level. The advantage to the HA in this case is that capacity decisions in a preventative healthcare service can be made based on feasible forecasts of future demand. Additionally, within the application domain, exploration of future sources of demand can be used to inform service planning.

The paper is organized as follows. Section 2 presents an overview of the use of demand and capacity support in healthcare, and reports on existing studies using a HA. Section 3 describes the HA, and Section 4 reports on the case study, data sources, the methodology and the forecasting results. Section 5 describes the HA DES results. Section 6 critiques this approach and concludes by summarizing the findings.

\section{LITERATURE REVIEW}

Given that the demand for health services is increasing and, for most public healthcare systems at least, they continue to operate in an environment of growing capacity and financial constraints, forecasting demand for a service can assist with optimal capacity planning and allocation of resources. Forecasting involves the prediction of future events based on acquiring foreknowledge using quantitative approaches or through estimation (Soyiri and Reidpath 2013). In healthcare, these methods have been used to forecast demand for services (Culliford et al. 2015; Diaz-Hierro 2012), investigate changes in disease profiles (Bebbington and Furniss 2014) and for predicting resource requirements (Worrall and Chaussalet 2015). However, the application of forecasting remains relatively undeveloped in health compared with other fields (Soyiri and Reidpath 2013). With increasing volumes and quality of both patient-specific and operational-level data being collected, an opportunity exists for the wider application of classical forecasting techniques (e.g., time-series models like moving averages, exponential smoothing) or, as is proposed in the paper, a HA combining forecasting with M\&S. The remainder of the literature review will focus on the latter.

Time-series analysis methods, based on decomposition of a data series and causal modeling, can integrate historical data patterns, including trends and seasonal effects, but may ignore other variables which can be predictors of demand (Diaz-Hierro 2012). Most forecasting utilizes historical data, but this comes with certain assumptions, namely that future patterns of demand for health services will replicate historical demand. Additionally, Worrall and Chaussalet (2015) argued that healthcare is dynamic by nature, and reforms in policy and scope mean that modellers have the dilemma of taking into account the influences of past changes on increasingly limited intervals of historical data when making projections. For example, Mielczarek (2013) highlighted misleading historical data in her demand model due to changes in the Polish healthcare system over recent years. Frequent and significant changes both internally and externally mean that historical data needs to be scrutinized to ensure that it can yield relevant information without distorting the forecasting results; yet data from a shorter time period lowers the degree of mathematical certainty (Cote and Tucker 2001). Along with this inevitable tension, it is widely recognized that the demand for healthcare is influenced by population changes in age and gender demographic profiles (Lowthian et al. 2011; Culliford et al. 2015).

Official population projections have been used in long-term healthcare forecasting to support strategic planning, with demographic shifts proposed as key drivers of demand (Lowthian et al. 2011; Strunk et al. 2006; Etzioni et al. 2003; Mielczarek 2013). For example, Jagger et al. (2006) predicted that ageing of the population alone, without accounting for disease prevalence or age-specific rates of disease, would result in a dramatic increase in disabled older people over a twenty-year period. Nonetheless, Wister and Speechley (2015) cautioned against using too simple an approach to forecasting healthcare demand, citing Angus (1987), who grossly underestimated overall US healthcare demand by assuming population change 
would be the only factor. Culliford et al. (2015) used an extrapolation model adjusted for population growth, compared with a static estimated rate applied to population forecasts, to forecast demand for jointreplacement surgery in England over ten years, and concluded that the extrapolation of historical demand gave estimates that were neither plausible nor sustainable, as compared to the static-rate model using demographics alone. Strunk et al. (2006) and Etzioni et al. (2003) used static-rate models and official population projections to forecast surgical and inpatient demand respectively across the USA, both concluding that specialties in which older patients constituted a greater share of work predicted larger forecasted workloads. Strunk et al. (2006) highlighted sharp regional contrasts in their findings; Cardosa et al. (2012) similarly asserted that health needs and demographic profiles are unique to locality, stressing the importance of this in operational planning. Additionally, Soyiri and Reidpath (2013) suggested that healthcare providers might be better informed and prepared with condition-specific health forecasts.

For capacity planning, simulation has been widely used in healthcare, and is a useful management tool for evaluating the impact of interventions without risk to the real system, and for comparing a wide range of outcomes. Efficiency of delivery of healthcare services relies on the coordination of demand and capacity, and simulation is often used to tackle these issues. For instance, Brailsford et al. (2009) in their analysis of the literature on simulation modeling in healthcare, found that simulation was used in $34 \%$ of papers classified by functional area as 'patient care requirement profiles and delivery planning'.

The most widely used simulation approach in heatlhcare is DES, as it is particularly useful for systems with a strong queueing structure and competition for resources (Gunal 2012). This is beneficial in hospitals where processes are dynamic and stochastic, hence DES was chosen as a suitable simulation method to combine with forecasting in this case study. DES has been used for endoscopy services for efficiency improvements at the operational level in several studies (Rex et al. 2005; Centano et al. 2010; Taheri et al. 2012). Pilgrim et al. (2009) used DES to model the bowel cancer patient pathway to examine the potential cost effectiveness of interventions. This highlighted the need for balanced decisions about investment in services, accounting for impacts on the rest of the system and demonstrating coupled relationships between different parts of the system. Similarly, an endoscopy referral pathway starting with demand for endoscopic investigation for each procedure, and incorporating targets for the rest of the pathway can capture knockon effects of delays on downstream targets in endoscopy appointments.

DES has had limited application in combination with forecasting approaches in healthcare. Mielczarek and Zabawa (2016) used demographic profiles with SD and DES to model the influence of population change on the demand for health services, but not the capacity perspective. Demir, Gunal and Southern (2017) used time-series forecasts of hospital admission data by specialty, which were used as inputs in a DES model; outputs were the key performance metrics over a period of time, across the whole hospital. A DES decision-support tool was developed by Mielczarek and Uzialko-Mydlikowska (2012) using a MonteCarlo model to simulate forecasts of emergency demand. This examined the influence of observed demographic trends on emergency demand as a forecasted DES input simulating patient pathways to estimate future healthcare service needs and budget for emergency care. A similar methodology was used by Mielczarek (2013) to simulate the volume and costs of emergency treatment. Arguably some individual or groups of conditions are strong predictors of ED attendance. For example Ceglowski et al. (2007) applied a data-driven 'casemix' approach to their simulation model to group similar patient conditions, modeling service demand for planning by diagnosis or diagnosis-group, rather than overall demand. These models looked at a high level of aggregation of conditions and treatments but there may be advantages to studying demand and capacity by individual service and procedure.

\section{HYBRID SYSTEMS MODELING APPROACH USING FORECASTING AND DES}

Eldabi et al. (2016) proposed a set of components used within a hybrid framework, noting that while some attempts have been made to develop hybrid frameworks for model development at both conceptual and implementation phases, most fail to condense all modeling steps into a simple, unified approach. A framework proposed by Zulkepli and Eldabi (2015) attempted to guide model development using a 3-phase 
framework: conceptualization, modeling, and communication. The resultant framework comprehensively demonstrated hybridization of the model logic as well as integration at the coding level, but focuses on hybrid simulation alone. Powell and Mustafee (2016) conceptualized a representation for a HA identifying a number of interdisciplinary methods that can be used in various stages of a HA study, and is a suitable, general, framework for approaching HA studies.

DES frequently uses inputs based on historical data to model a system. However when demand for a service has been increasing year-on-year through demographic shifts, policy change and deliberate attempts to improve population-level health (Brown et al. 2015), capacity planning tools should reflect this. DES can incorporate this in a hybrid approach using forecasting, to study the consequences of expected or theoretically feasible changes in demand on the system, rather than using estimates.

Demographic trends in most developed countries follow a similar pattern in terms of lower birth-rates, and a gradual ageing of the population (de Meijer et al. 2013). The UK population is predicted to rise to 69-million by mid-2024, an increase of 7.5\% (ONS 2016b) with a faster rate of growth than predicted two years ago; while the median age is rising such that by mid-2039, more than one-in-twelve of the population is projected to be aged 80 or over (ONS 2016b). Not only are the absolute numbers of older people growing, but with medical advances and improved standards of living, people are living longer. For these reasons, isolating the effect of demographic shifts on demand for a specific service can provide baseline evidence for decision-making. In the UK, the Office of National Statistics (ONS) provides subnational population projections to indicate the future size and age structure of the populations of English regions, local authorities and Clinical Commissioning Groups (CCGs). CCGs form the lowest level of health geography hierarchy in England. They are clinical-led groups representing all local GP practices, who are responsibility for buying and developing health services for local patients. However demographic change will not be the only contributor to future demand; incorporating historical patterns of demand can capture future demand patterns.

For this case study, the HA conceptual model (refer to section 5) uses forecasting scenarios for one diagnostic service and three procedures, to provide input data to simulation modeling for local service planning over a ten-year period. It uses both official population projections at the regional level, and historical demand data. This assists stakeholders to explore an understanding of future changes in demand, and how these sources of demand can influence capacity requirements and resource allocation decisions.

\section{CASE STUDY}

\subsection{Background}

Gastrointestinal (GI) endoscopy describes a set of procedures for diagnosing cancers of the stomach and esophagus (upper GI), bowel cancer (lower GI) and other conditions of the digestive tract. In the UK NHS, endoscopic investigations can be requested by General Practitioners (GP) via an urgent two-week (2WW) referral for suspected cancer, or the routine six-week pathway (6WW) as NHS-measured maximum wait times. New referral guidelines were launched in June 2015 (NICE 2015), increasing the sensitivity of GP referral criteria for endoscopy. Within the guidelines for upper GI referrals, provision is made for nonurgent referrals for upper endoscopy, where presentation criteria suggest a lower risk of cancer. In some parts of the UK, this has been interpreted as a four-week wait (4WW), measured within the 6WW target. The ongoing surveillance of high-risk groups is performed in conjunction with national guidelines. These include patients with a history of cancer, inflammatory disease or a genetic predisposition. Delays in the 2WW pathway have knock-on effects on further NHS targets. Evidence suggests that patients on surveillance programs are experiencing unacceptable delays due to delays within urgent two-week pathways (Brown et al. 2015). Those on $4 \mathrm{WW}$ pathways are likely to delay $6 \mathrm{WW}$ routine referrals, and cancer diagnoses within these referral pathways will have downstream consequences.

A set of public awareness campaigns between 2012-2015 (Cancer Research UK 2016) further contributed to increased endoscopy demand by encouraging early presentation and diagnosis. Endoscopic 
demand is both age- and gender-related. Cancer is primarily a disease of the elderly, with approximately $12 \%$ of the population aged over 70 years accounting for more than half of the total cancer diagnoses in 2014 (ONS 2014). Hence demand is regionally-specific, as population profiles vary. Torbay and South Devon NHS Foundation Trust (TSDFT) serves a resident population of approximately 375,000 people, with most of their endoscopy services commissioned from Torbay and South Devon Clinical Commissioning Group (TSDCCG). The Torbay area of Devon is attractive for retirement, so the proportion of those aged over 75 years, at 11\%, is higher than the UK national average of $8 \%$ (ONS 2016a, 2016 b).

\subsection{Data Sets}

ONS subnational projections for TSDCCG are used for baseline forecasts (ONS 2016b); older datasets are used for regression modeling in half years. This incorporates the first half of 2016, providing 8 data points (ONS, 2012, 2013, 2014). Note that the base year is two years previous to the release year, so the 2016 release contains projections from 2014. CCGs were created in 2013, so the data available for CCG-level population prior to this was back-casted from the 2011 census (ONS 2013, 2014) and has discrepancies.

For short-term validation of ONS projections, data from NHS Digital/Health and Social Care Information Centre (HSCIC) was used. The dataset is 'Number of Patients Registered at a GP practice by CCG', at April 2016. This is a quarterly snapshot of the number of patients per GP practice per CCG in England (HSCIC/NHS Digital 2016).

Anonymized hospital demand data was extracted from the patient administration system from June 2011 to June 2016. While a second set of data from 2001 was obtained to compare overall demand with population, no usable data was stored before 2009. The data was filtered to contain three procedures: colonoscopy, upper OGD, and sigmoidoscopy. A primary assumption in this method is that only patients within the TSDCCG are referred into TSDFT; at more than $99.33 \%$ this assumption is deemed valid.

\subsection{Forecasting methods}

Short-term subnational ONS projection for a small geographic area was validated, as these are considered to be less reliable than larger geographic areas (ONS 2015). HSCIC/NHS Digital (2016) data by 5-year age-group and gender was compared with ONS subnational population projections for 2016 by age-group and gender in TSDCCG. This assumes that all persons within the TSDCCG boundaries are registered with a GP practice, and acts as a proxy for accurate population count per age-group and gender for mid-2016. In the short-term, the ONS projections were found to have acceptable accuracy.

Historical data from 2012 was disaggregated into three procedures, both genders, and five-year age groups. Patients presenting as part of the national bowel-cancer screening program were removed from the dataset. Patients undergoing surveillance; and those deceased or removed from the waiting list were analyzed separately. Percentage-changes for each year of the 2016 population projections were calculated by age-group and gender to 2026 and applied to hospital demand age-gender cohorts at 2015. Projections for each half-year were based on the previous actual half-year period. This was primarily to include Januaryto-June 2016 demand data. Additionally, for some procedures, differences in half-years were observed. The data was then re-aggregated to give demand per procedure, with a static base rate of demand at 2015. This baseline assumes that the rate of demand per age-gender cohort will remain static or in line with projected population rates, isolating the effect of population growth and ageing on endoscopy demand.

Historical proportion of demand relative to the population was calculated for each half year from 20122016 (8 data points). Using the disaggregated data, for each procedure and age-group per year, a regression of demand as a percentage per unit population, and year, was calculated and projected to 2026 (see Figure 1). This was adjusted as a proportion of the 2015 demand rate, which was already accounted for in the population projection forecasts. These adjusted figures were multiplied by the baseline demand counts for 2016 onwards then re-aggregated to give projections per age-group, procedure and year. This gave forecasts 
that used both ONS population projections and historical demand per procedure. This method was validated using SPSS 23.0 Time-Series Modeler (Section 4.5).

\begin{tabular}{|c|c|c|c|c|}
\hline UpperOGD & 65-69 year & & & \\
\hline Year & Baseline & \%pop/year & Adjustment & Forecast \\
\hline 2012 & 155 & 0.75242718 & & \\
\hline 2013 & 180 & 0.88235294 & & \\
\hline 2014 & 209 & 1.03974927 & & \\
\hline 2015 & 199 & 1.01461323 & & \\
\hline 2016 & 191.8415 & 0.99107869 & 0.97680442 & 187.3917 \\
\hline 2017 & 186.3654 & 1.11891325 & 1.10279781 & 205.5234 \\
\hline 2018 & 181.6942 & 1.13667649 & 1.12030521 & 203.553 \\
\hline 2019 & 175.4463 & 1.14965253 & 1.13309435 & 198.7972 \\
\hline 2020 & 168.191 & 1.20688975 & 1.18950721 & 200.0644 \\
\hline 2021 & 159.9837 & 1.25935056 & 1.24121243 & 198.5737 \\
\hline 2022 & 154.0589 & 1.27962288 & 1.26119278 & 194.2979 \\
\hline 2023 & 148.7906 & 1.32511569 & 1.30603036 & 194.325 \\
\hline 2024 & 147.723 & 1.37122412 & 1.3514747 & 199.6439 \\
\hline 2025 & 149.7748 & 1.40677076 & 1.38650937 & 207.6642 \\
\hline 2026 & 152.7516 & 1.44434929 & 1.42354667 & 217.4491 \\
\hline
\end{tabular}

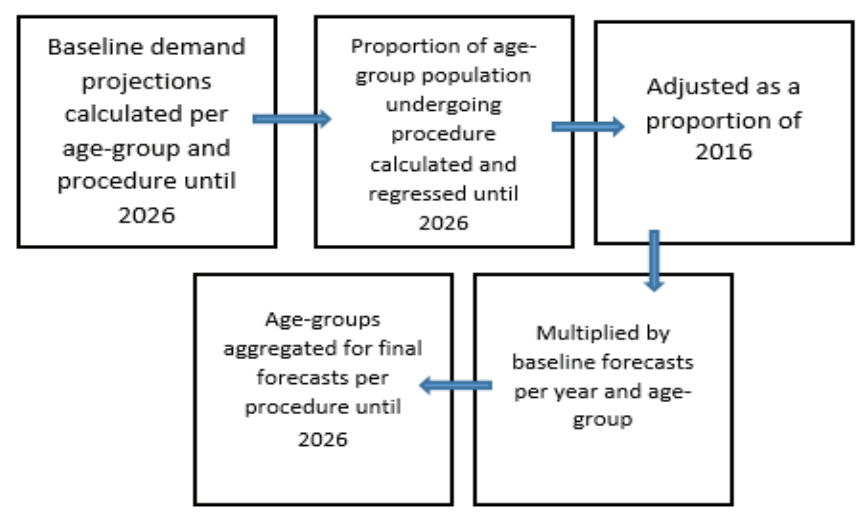

Figure 1: Subset of data for one procedure and single age-group with flow-chart of method.

\subsection{Analysis and Results}

Historical hospital data shows an increase in overall demand from roughly 6000 procedures per year in 2009, to nearly 9000 in 2015, with females consistently higher users. Historical demand overall is strongly age-related. This is seen in all procedures and all years. The highest demand comes from those aged 55-80 years. With the ONS data, a largely steady overall increase in population is seen in the TSDCCG area from 2009 to 2015. The highest proportion of population increase is projected to be between the ages of 71-80 years. A steady linear increase in the proportion of overall demand (\%) relative to the population is seen, from $2.09 \%$ in 2009 to $3.16 \%$ in 2015 .

Using the method described to create the baseline forecasts based on demographic change, the demand as a percentage of population is relatively stable. This is because the forecast is projected using population projections from a static historical rate of demand in 2015/16. The rate of projected population change (ONS 2016a, base year 2014) is slow, increasing over ten years from $0.52 \%$ per year to $0.55 \%$ per year. However the baseline projected demand is expected to outpace the rate of population growth (Figure 2). This is because a disproportionate number of older patients use the service.

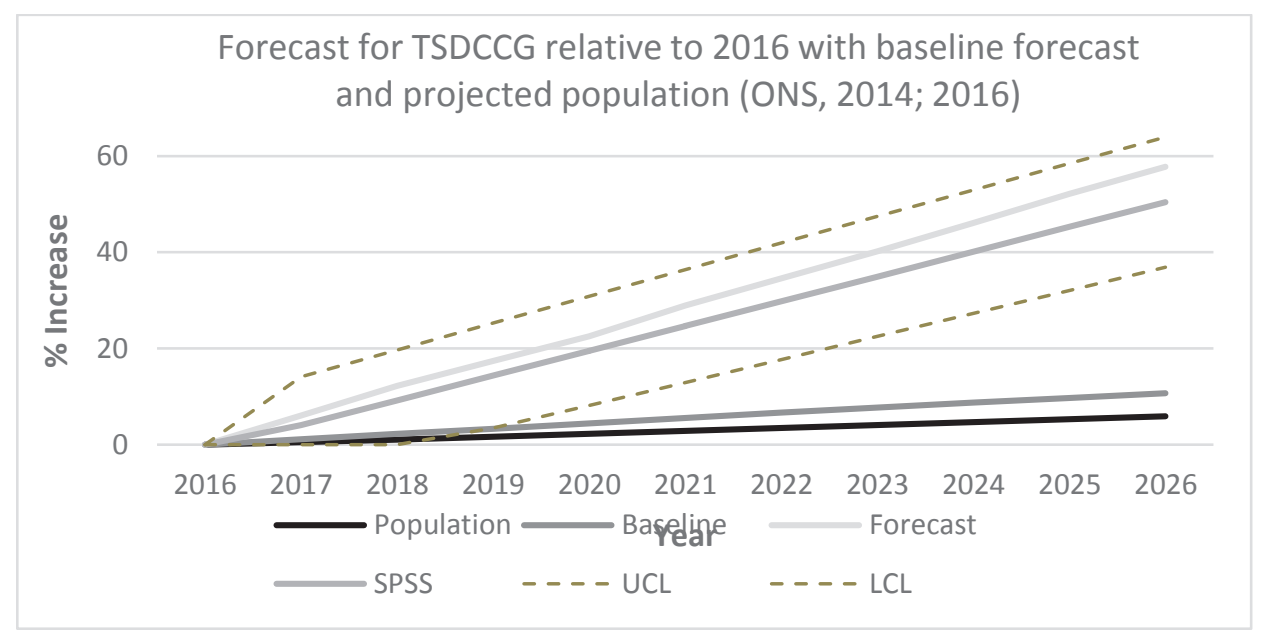

Figure 2: Combined forecast, SPSS validation with UCL and LCL, baseline forecast (demographic change only), and actual population projection TSD (ONS 2014, 2016), adjusted relative to 2016. 
The final forecasts by individual procedure over a ten-year period were calculated. These forecasts account for both demographic change, and using historic data, other factors influencing demand, including future policy changes to increase early diagnosis. For individual procedures, this method forecasts an increase over ten years in Upper OGD demand of 71\%; for Colonoscopy a $40 \%$ increase; and for Sigmoidoscopy a 65\% increase from 2016 (Figure 2). In 2026, demand for endoscopy services overall is predicted to rise $58 \%$ from 2016 levels, with $3.9 \%$ of the projected population undergoing endoscopy, compared to $2.2 \%$ in 2012 .

\subsection{Validation}

The overall (combined) forecast was validated against SPSS 23.0 Time Series Modeler, using both actual counts (Figure 3) and proportion of the population using endoscopy services, from 2012-2016. The combined forecasted results as described in Section 4.4 fall within the calculated 95\% CI's for actual counts and for percentage of projected population undergoing endoscopy, for all years 2017-2026 (Table 1). SPSS predicts a $50 \%$ increase in demand over ten years, whereas the combined forecast adjusted for demographic change predicts a $58 \%$ increase in demand by 2026 .

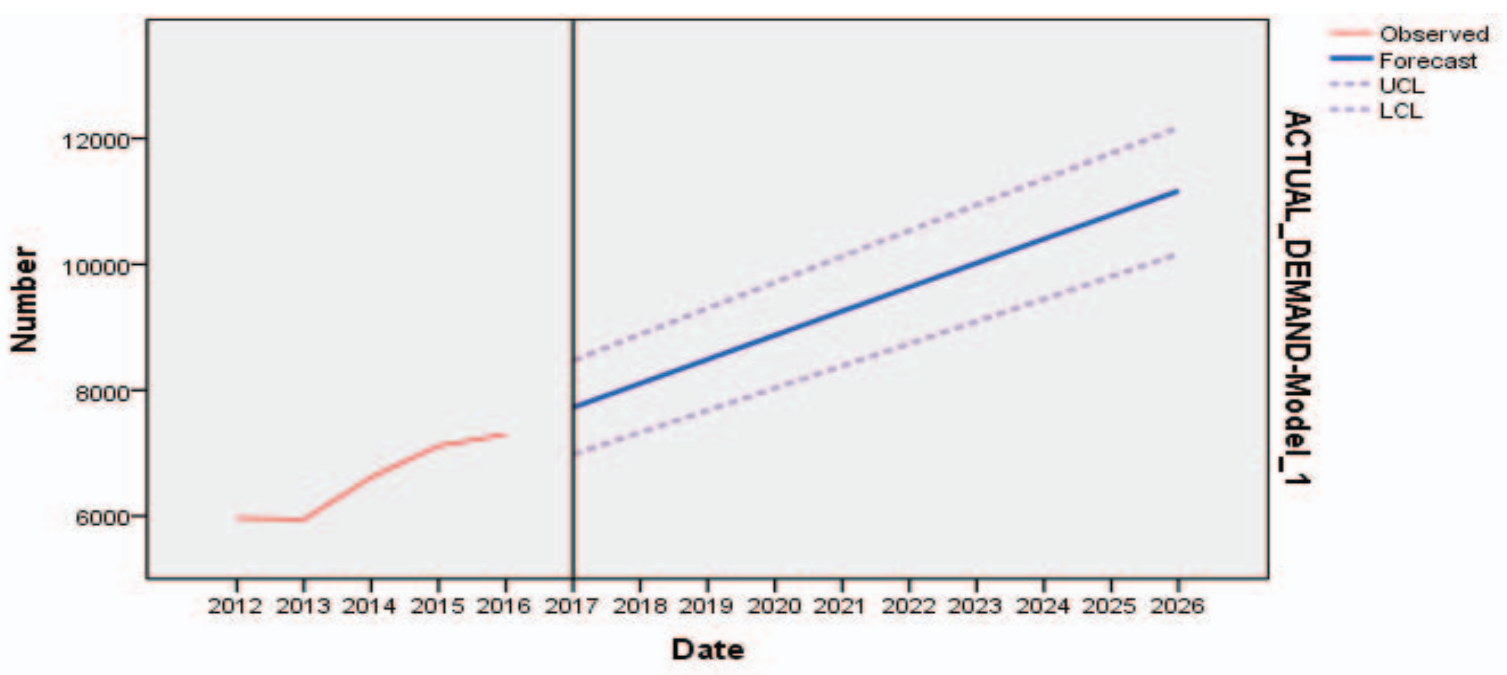

Figure 3: Combined procedure SPSS time-series forecast by actual counts 2016-2026 (95\%CI).

Table 1: Forecasts (actual counts of combined procedures) compared with forecasted demand of actual counts (combined procedures) using SPSS time-series modeler with UCL and LCL (95\% CI).

\begin{tabular}{|l|c|c|c|c|c|c|c|c|c|c|}
\hline MODEL & $\mathbf{2 0 1 7}$ & $\mathbf{2 0 1 8}$ & $\mathbf{2 0 1 9}$ & $\mathbf{2 0 2 0}$ & $\mathbf{2 0 2 1}$ & $\mathbf{2 0 2 2}$ & $\mathbf{2 0 2 3}$ & $\mathbf{2 0 2 4}$ & $\mathbf{2 0 2 5}$ & $\mathbf{2 0 2 6}$ \\
\hline Forecast & 7874 & 8331 & 8716 & 9100 & 9575 & 9990 & 10408 & 10846 & 11296 & 11716 \\
\hline SPSS Forecast & 7726 & 8108 & 8491 & 8874 & 9256 & 9639 & 10021 & 10404 & 10787 & 11169 \\
\hline UCL & 8472 & 8887 & 9302 & 9715 & 10127 & 10538 & 10948 & 11357 & 11766 & 12174 \\
\hline LCL & 6980 & 7329 & 7680 & 8032 & 8386 & 8740 & 9095 & 9451 & 9808 & 10165 \\
\hline
\end{tabular}

\section{DES MODEL WITH DEMAND FORECAST}

The DES model, implemented in Simul8 (https://www.simul8.com) (see Figure 4), is being used in this ongoing study for the stakeholder objective of understanding the downstream effects of expected year-on- 
year increases in demand for endoscopic procedures. Two models have been implemented - the Stable Demand Model (SDM) and the Forecasted Demand Model (FDM). We have developed SDM as a representation of conventional models that do not take into account the expected changes in demand over time, against a more dynamic FDM which uses forecasted demand to switch input distributions at runtime. We assert that the latter is a better representation of reality. The objective of SDM is, thus, to compare simulation results with FDM, and thereby demonstrate the utility of the HA approach. Once this had been achieved we propose to use the FDM DES model to realize the following three stakeholder objectives: (i) to investigate the effects on the current system of expected annual increases in demand upon the system; (ii) to investigate the effects of changes to the distribution of 2, 4 and 6 week referrals per procedure; and (iii) to experiment with alternative capacity management strategies with the objective of proactive service planning toward the projected increases in demand. This is ongoing work and we expect to present the results of the DES study in a future publication.

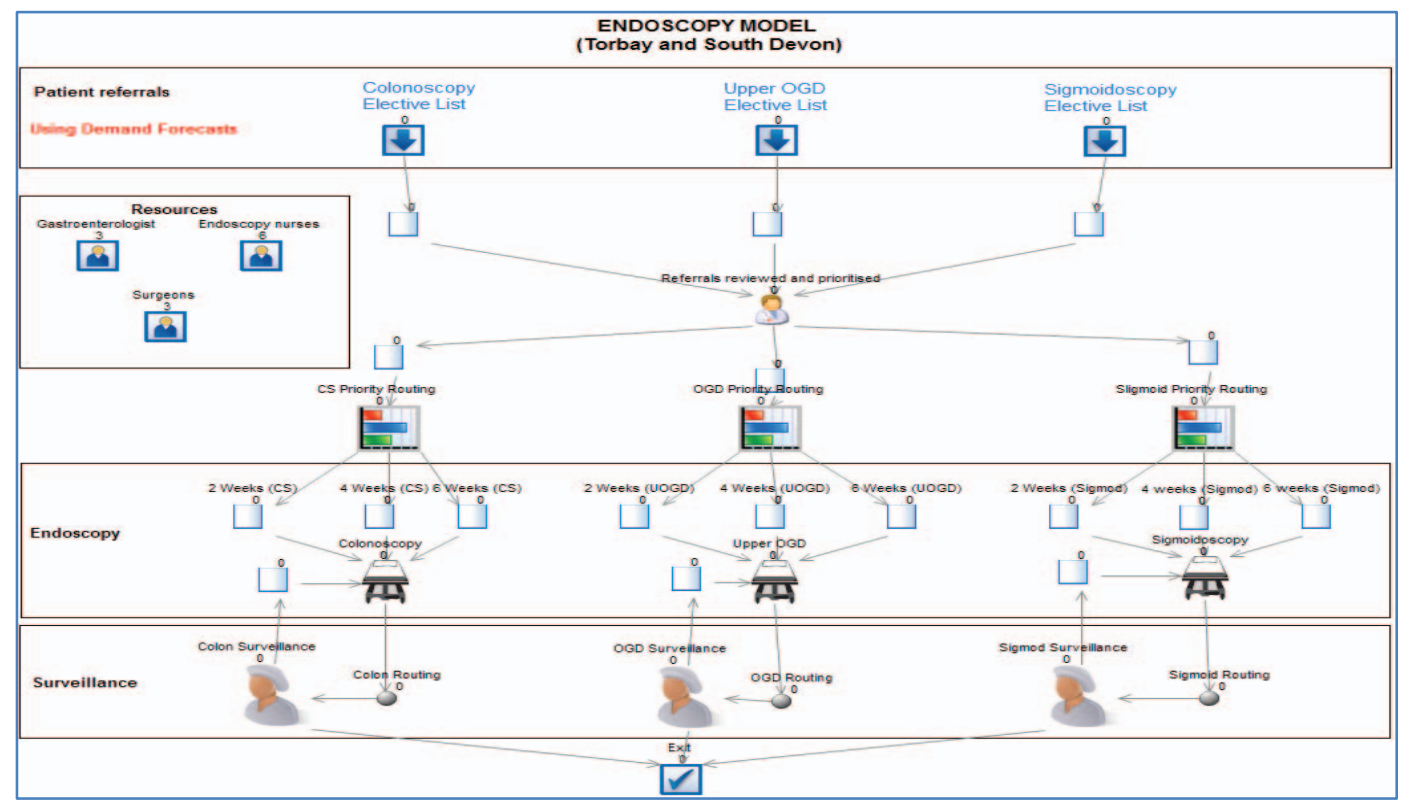

Figure 4: Modeling patient referrals into endoscopy using DES - The FDM model.

Both SDM and FDM generate GP patient referrals for each of the three procedures (colonoscopy, upper OGD and sigmoidoscopy) and follow the same workflow (Figure 4). Within the model, every patient referral is reviewed by a consultant and assigned a value for a 2 week, 4 week or 6 week wait as indicated by the urgency of their referral. Our implementation allows investigation of the effects of altering the balance of 2, 4 and 6 week referral patterns, as near-future policies suggest an increase in urgent $2 \mathrm{WW}$ appointments for early diagnosis, and the possibility of all appointments using a $4 \mathrm{WW}$ target. In addition, numbers of patients re-entering the system for surveillance can be investigated; due to changes in patient screening, these proportions are expected to increase. The DES has three procedure rooms - a preliminary assumption of this model is that each procedure is allocated identical daily time. Triangular distributions define the time for each procedure, including turn-around time. The model runs for two, four-hour sessions per day, five days per week.

SDM and FDM differ in relation to the algorithm used for the generation of GP patient referrals in the model. In the case of SDM, the input distributions were the demand patterns calculated based on historic data (hospital demand data from June 2011-June 2016). For FDM the distributions were based on forecasted demand (see section 4.3 and Figure 5) and discrete distributions were fitted for each procedure/year (3 procedures over eight-year period, with a 1-year warm-up, a total of 24 Poisson 
distributions). We compared the simulated datasets with the forecasting models and verified that the FDM was returning acceptable ranges of data from these distributions.

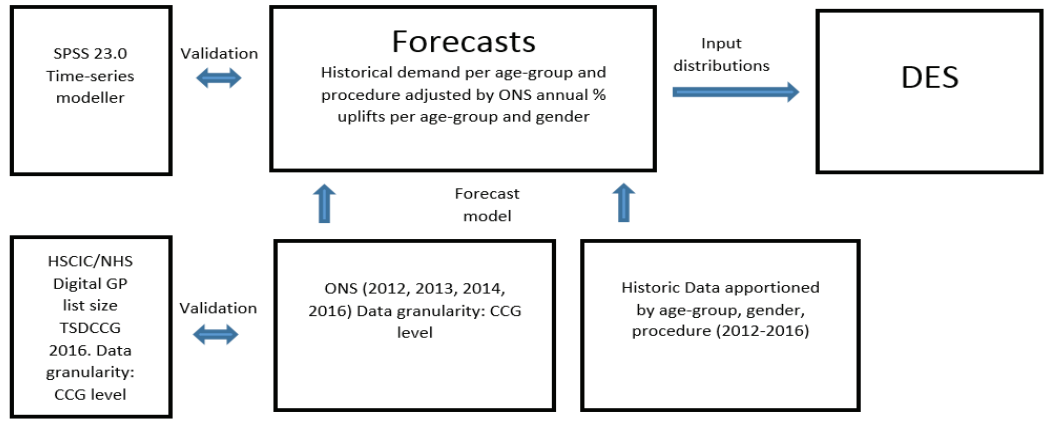

Figure 5: HA conceptualization for FDM.

The preliminary results of the DES models are being used for the process of model verification and calibration. The numbers of patient referrals generated by SDM and FDM until 2022 can be seen in Figure 6. The SDM model using current demand generates stable figures; the FDM model, which updates its parameters annually with reference to the forecasts (this is implemented using Visual Logic), shows a steady increase in demand for each procedure that reflects those generated by the forecasts.

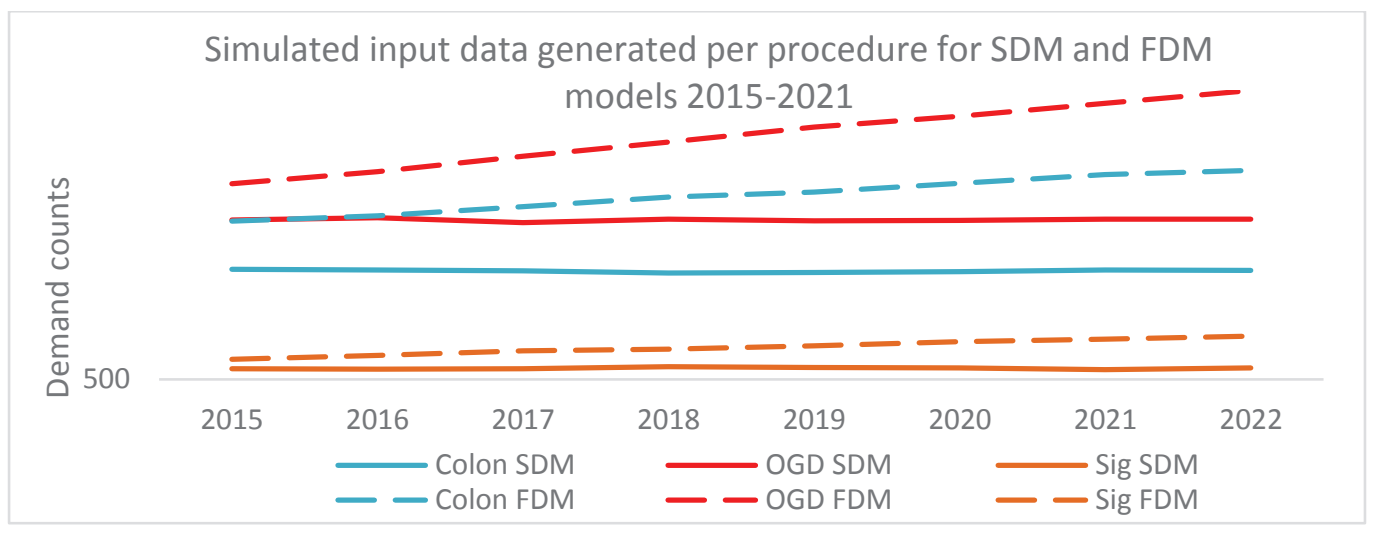

Figure 6: Patient referrals generated by DES by the static model; and by the forecast model.

\section{CONCLUSION}

A reliable health forecast is important to enhance preventive health service delivery and support decisionmaking for optimized allocation of scare resources. Most healthcare forecasting models predict demand without regard for future capacity constraints. DES is a suitable method for testing different scenarios involving service and capacity changes, given information about demand for a system. Efficiency improvements in healthcare can be gained by improving the match between demand and capacity, and modeling pathways as a function of demand factors, using a combination of forecasting and DES. There has been limited application of hybrid-systems modeling approaches using forecasting with DES in healthcare, however no single model can adequately capture both dimensions. A HA has the potential to realize synergies across techniques and enable greater insights into a problem situation. The case study demonstrates a hybrid approach, the aim of which is to demonstrate the effect of future changes in demand for individual diagnostic procedures on a preventative healthcare service. Making valid predictions of future demand supports decision making for capacity planning and resource allocation; this is done by attempting to link demographic trends locally with local volume of demand for services, by combining 
different data sources. These forecasts are then used in a DES model which updates its input distributions annually. This assumes that valid forecasts can be generated from projected demographic trends and historical demand for a service, which is considered plausible. There is also the assumption that the proportion of future use of services will remain stable per age-group. Given that demand beyond demographic change is driven mostly by policy for this service, this is considered an acceptable assumption, and a more realistic scenario for capacity planning than the use of stable distributions over time.

\section{REFERENCES}

Bebbington, E., and D. Furniss. 2014. "Linear Regression Analysis of Hospital Episode Statistics Predicts a Large Increase in Demand for Elective Hand Surgery in England". Journal of Plastic Reconstruction, \& Aesthetic Surgery 68(2):243-251.

Brailsford, S., P. R. Harper, B. Patel, and M. Pitt. 2009. "An Analysis of the Academic Literature on Simulation and Modeling in Health Care". Journal of Simulation 3(3):130-140.

Brown, H., S. Wyatt, S. Croft, N. Gale, N. Turner, and A. Mulla. 2015. Scoping the Future: An Evaluation of Endoscopy Capacity Across the NHS in England. Commissioned by Cancer Research UK, 2015.

Cardosa, T., M. D. Oliveira, A. Barbosa-Povoa, and S. Nickel. 2012. "Modeling the Demand for LongTerm Care Services Under Uncertain Information”. Health Care Management Sciences 15(4):385-412.

Ceglowski, A., L. Churilov, and J. Wassertheil. 2007. "Combining Data Mining and Discrete Event Simulation For a Value-Added View of a Hospital Emergency Department". Journal of the Operational Research Society 58(2):246 - 254.

Centano, M., H. Dodd, M. Aranda, and Y. Sanchez. 2010. "A Simulation Study to Increase Throughput in an Endoscopy Centre". In Proceedings of the 2010 Winter Simulation Conference, edited by B. Johansson, S. Jain, J. Montoya-Torres, J. Hugan, and E. Yücesan, 2462-247. Piscataway, New Jersey: IEEE.

Cote, M., and S. Tucker. 2001. "Four Methodologies to Improve Healthcare Demand Forecasting". Healthcare Financial Management 55(5):54-58.

Culliford, D., J. Maskell, A. Judge, C. Cooper, D. Prieto-Alhambra, and N. Arden. 2015. "Future Projections of Total Hip and Knee Arthroplasty in the UK: Results From the UK Clinical Practice Research Datalink". Osteoarthritis and Cartilage 23(4):594-600.

Demir, E., M. Gunal., and D. Southern. 2017. "Demand and Capacity Modeling for Acute Services Using Discrete Event Simulation”. Health Systems 6(1):33-40.

De Meijer, C., B. Wouterse, J. Polder, and M. Koopmanschap. 2013. "The Effect of Population Ageing on Health Expenditure Growth: A Critical Review". European Journal of Ageing 10(4):353-361.

Diaz-Hierro, J., J. J. Martin, A. V. Arenas, M. P. Gonzalez, J. M. Arevalo, and C. V. Gonzalez. 2012. "Evaluation of Time-Series Models for Forecasting Demand for Emergency Health Care Services". Emergencias 24:181-188.

Eldabi, T., M. Balaban, S. Brailsford, N. Mustafee, R. Nance, S. Onggo, and R. Sargent. 2016. "Hybrid Simulation: Historical Lessons, Present Challenges, and Futures". In Proceedings of the 2016 Winter Simulation Conference, edited by T. M. K. Roeder, P. Frazier, R. Szechtman, E. Zhou, T. Huschka, and S. Chick. 1388-1400. Piscataway, New Jersey: IEEE.

Etzioni, D., J. Liu, M. Maggard, and C. Ko. 2003. "The Ageing Population and its Impact on the Surgery Workforce”. Annals of Surgery 238(2):170-177.

Fakhimi, M., N. Mustafee, and L. Stergioulas. 2016. "An Investigation into Modeling and Simulation Approaches for Sustainable Operations Management”. Simulation, 92(10):907-919.

Gunal, M. 2012. “A Guide for Building Hospital Simulation Models". Health Systems 1(1):17-25.

Harper, P.R. 2002. "A Framework for Operational Modeling of Hospital Resources". Health Care Management Science 5(3):165-173. 
HSCIC/NHS Digital (2016) Number of Patients Registered at a GP Practice CCG, April 2016. NHS Digital. Access through http://content.digital.nhs.uk.

Jagger, C., R. Matthews, N. Spires, C. Brayne, A. Comas-Herrera, T. Robinson, J. Lindesay, and P.Croft. 2006 Compression or Expansion of Disability?: Forecasting Future Disability Levels Under Changing Patterns of Diseases. Kings Fund, London, UK.

Katsaliaki, K., and N. Mustafee. 2011. "Applications of Simulation Within the Healthcare Context". Journal of the Operational Research Society 62(8):1431-1451.

Lowthian, J., D. Jolley, A. Curtis, A. Currell, P. Cameron, J. Stoelwinder, and J. McNeil. 2011. “The Challenges of Population Ageing: Accelerating Demand for Emergency Ambulance Services by Older Patients, 1995-2015". Medical Journal of Australia 194(11):574-578

Mielczarek, B. 2013. "Estimating Future Demand for Hospital Emergency Services at the Regional Level". In Proceedings of the 2013 Winter Simulation Conference, edited by R. Pasupathy, S.-H. Kim, A. Tolk, R. Hill, and M. E. Kuhl, 2386-2397. Piscataway, New Jersey: IEEE.

Mielczarek, B., and B. Uzialko-Mydlikowska. 2012. "Using Simulation to Forecast the Demand for Hospital Emergency Services at the Regional Level". In Proceedings of the 2012 Winter Simulation Conference, edited by C. Laroque, J. Himmelspach, R. Pasupathy, O. Rose, and A. M. Uhrmacher, 2 pages. Piscataway, New Jersey: IEEE.

Mielczarek, B., and J. Zabawa, 2016. "Modeling Healthcare Demand using a Hybrid Simulation Approach". In Proceedings of the 2016 Winter Simulation Conference, edited by T. M. K. Roeder, P. I. Frazier, R. Szechtman, E. Zhou, T. Huschka, and S. E. Chick, 1535-1546. Piscataway, New Jersey: IEEE

NICE. 2015. Suspected Cancer: Recognition and Referral, In NICE Guidelines.

ONS. 2016a. Subnational Population Projections, Clinical Commissioning Groups in England: Z2. Office of National Statistics. Access through http://www.ons.gov.uk.

ONS. 2016b. National Population Projections: 2014-based Statistical Bulletin. Office of National Statistics. Access through http://www.ons.gov.uk.

ONS. 2015. Subnational Population Projections Accuracy Report, August 2015. Office of National Statistics. Access through http://www.ons.gov.uk.

ONS. 2014. Clinical Commissioning Group Mid-year Population Estimates. Office of National Statistics. Access through http://www.ons.gov.uk.

ONS. 2013. Mid-2002 to Mid-2010 Population Estimates for Clinical Commissioning Groups (CCGs) in England by Single Age and Sex Based on the Results of the 2011 Census. Office of National Statistics. Access through http://www.ons.gov.uk.

Pilgrim, H., P. Tappenden, J. Chilcott, M. Bending, P.Trueman, A. Shorthouse, and J. Tappenden. 2009. "The Costs and Benefits of Bowel Cancer Service Developments Using Discrete Event Simulation." Journal of the Operational Research Society 60(10):1305-1314.

Powell, J. H., and N. Mustafee. 2016. "Widening Requirements Capture with Soft Methods: An Investigation of Hybrid M\&S Studies in Healthcare." Journal of the Operational Research Society. http://rdcu.be/n4ov (online first).

Rex, D., B. Lahue, R., Dronzek, and M. Lacey. 2005. "Impact of Two Procedure Rooms Per Physician on Productivity: Computer Simulation Examines the Impact of Process Change in the Hospital Gastroenterology Department". Gastrointestinal Endoscopy 61(5):154-159.

Soyiri, I., and D. Reidpath. 2013. "An Overview of Health Forecasting". Environmental Health and Preventative Medicine 18(1):1-9.

Strunk, B., P. Ginsburg, and M. Banker. 2006. "The Effect of Population Ageing on Future Hospital Demand". Health Affairs 25(3):141-149.

Taheri, J., Z. Gellad, D. Burchfield, and K. Cooper. 2012. "A Simulation Study to Reduce Nurse Overtime and Improve Patient Flow Time at a Hospital Endoscopy Unit". In Proceedings of the 2012 Winter 
Simulation Conference, edited by C. Laroque, J. Himmelspach, R. Pasupathy, O. Rose, and A. M. Uhrmacher, 1027-1038. Piscataway, New Jersey: IEEE.

Wister, A.. and M. Speechley. 2015. "Inherent Tensions Between Population Ageing and Healthcare Systems: What Might the Canadian Healthcare System Look Like in Twenty Years?" Population Ageing 8(4):227-243.

Worrall, P., and T. Chaussalet. 2015. "A Structured Review of Long-Term Care Demand Modelling". Health Care Management Science 18(2):173-194.

Zulkepli, J., and T. Eldabi. 2015. "Towards a Framework for Conceptual Model Hybridization in Healthcare". In Proceedings of the 2015 Winter Simulation Conference, edited by L. Yilmaz, W. K. V. Chan, I. Moon, T. M. K. Roeder, C. Macal, and M. D. Rossetti. 1597-1608. Piscataway, New Jersey: IEEE.

\section{AUTHOR BIOGRAPHIES}

ALISON HARPER is a PhD student at the University of Exeter Business School, UK with an interest in healthcare modeling and predictive analytics. Her email address is ah596@exeter.ac.uk.

NAVONIL MUSTAFEE is Senior Lecturer in Operations Management at the University of Exeter Business School, UK. He holds a MSc and Ph.D. in Distributed Computing and Simulation from Brunel University, UK. His research interests include hybrid systems modelling, modelling methodologies, business intelligence and predictive analytics, M\&S in healthcare, and bibliometric analysis. He is an Associate Editor for Simulation: Transactions of the SCS and the Journal of Simulation. His email address isn.mustafee@exeter.ac.uk.

MARK FEENEY is a consultant gastroenterologist and endoscopy lead at Torbay and South Devon NHS Foundation Trust. His email address is mark.feeney2@nhs.uk. 